\title{
Biological effects and cytotoxicity of tricalcium phosphate and formaldehyde cross-linked gelatin composite
}

\author{
Chun-Hsu Yao a, Jui-Sheng Sun ${ }^{b}$, Feng-Huei Lin ${ }^{c, *}$, Chun-Jen Liao ${ }^{d}$ \\ Chin-Wang Huang a \\ a Department of Chemistry, Chung Yuan Christian University, Chung-Li, Taiwan, ROC \\ 'Department of Orthopedic Surgery, National Taiwan University Hospital, Taipei, Taiwan, ROC \\ 'Center for Biomedical Engineering, College of Medicine, National Taiwan University, Taipei, Taiwan, ROC \\ ' Department of Materials Engineering, Tatung Institute of Technology, Taipei, Taiwan, ROC
}

Received 5 July 1995; accepted 28 August 1995

\begin{abstract}
The purpose of this study was to prepare and evaluate a composite, GTF, combining tricalcium phosphate (TCP) ceramic particles and formaldehyde cross-linked gelatin, as bone substitute. The content of formaldehyde in the developed material was used to control the physical and mechanical properties of the gelatin structure, in terms of cross-linked gelatin molecules, solubility, and biodegradation of the reconstituted matrices. Formaldehyde, however, is known to be a potentially toxic substance that would cause inflammation and severe tissue response. Thus, it is necessary to know whether the substances released from the GTF composite will cause severe tissue response. In this study, the biocompatibility and cytotoxicity of the GTF composite were examined by the in vitro method of human myoblast cell culture. Extracts were obtained by soaking the GTF composite in normal saline for $1,2,4,6$, and 8 days. The extract mixed with complete medium in a ratio of $1: 1$ was added to cell culture wells each containing $1 \times 10^{5}$ myoblasts. After being cultured for 2 days, the cells were trypsinized and counted in a Neubauer countingchamber under an optical microscope. The culture dishes were washed with $0.185 \mathrm{M}$ sodium cacodylate buffer ( $\mathrm{pH} 7.2$ ) and fixed with $4 \%$ glutaraldehyde solution for $30 \mathrm{~min}$. They were then dehydrated through a graded ethanol series and stained with hematoxylin/eosin for optical microscopic examination. The substances released from the GTF composite were analyzed by gas chromatography $(\mathrm{GC})$, capillary electrophoresis (CE), and inductive coupled plasma-atomic emission spectrometry (ICP-AES).

The results showed that the GTF composite was well tolerated by the myoblast. The unchained or uncross-linked formaldehyde was completely released from the GTF composites after being soaked in the normal saline for 4 days. The extract was thought to inhibit the cell growth for the initial 4 days of soaking time. After soaking for 4-6 days, the GTF composite gradually began to release some nutritious elements which were bencficial to the myoblast growth and caused cell numbers to increase substantially. The released nutritious constituents were analyzed by the above-mentioned instruments. The results were in agreement with the above observations.
\end{abstract}

Keywords: Bioabsorbed bone graft; Cytotoxicity; Tricalcium phosphate; Cross-linked gelatin; Myoblast

\section{Introduction}

Bone tissue damage, caused by trauma, tumor and infection, creates bone defects of various shapes and sizes which must be filled with a suitable substance to accelerate healing or enable them to heal at all. The function of a refilled biomaterial is to lead to both

\footnotetext{
* Corresponding author. Fax: +886 2 3940049; e-mail: double@bmec.mc.ntu.edu.tw
}

0254-0584/96/\$15.00 (C 1996 Elsevier Science S.A. All rights reserved SSDI 0254-0584(95) 01702-3 migration of bone cells toward and inside the biomaterial and the production of these induced cells to replace the implant and return the cavity to natural bone [1-3].

Since infection phenomena and the risk of viral transmission are frequent problems, transplantation of living tissue by means of allogenic or xenogenic grafts represents only a minor part of reconstructive surgery. Removal of the autogenic tissue creates additional surgical trauma, which also limits its medical application [3-6]. To overcome these problems of natural grafts, research 
on bone-substitute materials has been developed. However, the biocompatibility and mechanical stresses of synthetic materials have strictly limited the success of any artificial biomaterials $[7,8]$.

Clinically, bioceramics, such as HA, tricalcium phosphate (TCP) and bioglass, have been tried for use as bone implants. The use of these bioactive ceramics for defect filler was in powder and block forms. Although powder ceramics remain the form of choice for filling small irregular defects, the therapeutic effect of the filling implant was lost by migration of particles from the defect site. Furthermore, it was too difficult to handle and keep in place compactly for convenient fabrication and operation of block-type ceramics [911]. Thus, it is necessary to mix a suitable binder with the granular material to overcome these problems. Recently, composites of ceramic powders with natural degradable polymers have attracted much interest as a bone filler [12-15]. Fibrin, collagen and gelatin are generally considered as binders, as they have good adhesiveness and plasticity properties. In this study, a composite, GTF, combining formaldehyde cross-linked gelatin and TCP, was designed as a bone substitute. The weight ratio of TCP and formaldehyde cross-linked gelatin in the composite was $3: 1$, which was equivalent to that of human natural bone. In the composite, the formaldehyde cross-linked gelatin and TCP served as a continuous matrix (binding agent) and discontinuous particles, respectively. The presence of gelatin in the GTF composites serves two purposes [16,17]: it immobilizes the TCP particles and it improves the mechanical properties. While this composite does not form in vivo, it is resorbable. The gelatin matrix is resorbed, allowing for new bone intergrowth around the TCP particles. The TCP particles are also resorbable, but the resorption rate of these particles is much slower than that of the gelatin matrix, and is controlled by cellular and solution-driven processes. Gelatin is easily resorbable in vivo, the cross-linking agents (formaldehyde) being added to prolong its stay in the living tissue and improve the mechanical propertics of the composite [18-22].

Therefore, a mixture of TCP powder and gelatin treated with formaldehyde solution was prepared, and its possible use as a bone defect filler was evaluated. Although the formaldehyde cross-linked GTF composite reduces resorption and limits antigenicity of collagenous and noncollagenous proteins, and renders the materials less susceptible to bacterial and fungal invasion or destruction, the adverse effects of residual formaldehyde have to be considered and led to the present study. The study was focussed on the cytotoxic evaluation of the GTF composite. Human myoblast was prepared and cultured for testing the cytotoxicity of the GTF composite. The results obtained were in terms of formaldehyde release, gelatin concentration, calcium concentration, myoblast counting, and morphological or functional changes of the myoblast.

\section{Materials and methods}

\subsection{Material preparation}

- The TCP powder was provided by Ishizu Pharmaceutical Co., Ltd., Japan, with a chemical formula of $\mathrm{Ca}_{3}\left(\mathrm{PO}_{4}\right)_{2}$. The ceramic powder was placed in a platinum crucible and sintered in a $\mathrm{SiC}$-element furnace at a temperature of $1000^{\circ} \mathrm{C}$ for $1 \mathrm{~h}$, and then cooled down to room temperature. The sintered ceramic powder was crushed in an alumina grinding bowl and sieved in 40-60 mesh. The TCP ceramic particles were obtained for material preparation. The crystal structure of the TCP particles was in agreement with that of whitlockite (mineral name of TCP), and no other peaks appeared in the X-ray diffraction (XRD) pattern.

The gelatin used in the study was produced by Wako Pure Chemical Industries, Ltd., and was extracted from bovine skin with an average molecular weight of about 18000 . The gelatin powder was weighed and placed in a $1000 \mathrm{~cm}^{3}$ heaker which was then put into a water bath with a temperature of $75^{\circ} \mathrm{C}$. The gelatin solution was stirred and dissolved in the distilled water until a homogeneous $16.7 \%$ gelatin solution was attained. The sintered TCP ceramic particles were poured in and mixed with the gelatin solution, and stirred continuously at the same temperature all the time, in order to achieve a better distribution of ceramic particles in the gelatin matrix. A series of formaldehyde solutions in different concentrations were added to the ceramic/gelatin mixture for gelatin matrix cross-linking. After $15 \mathrm{~min}$ the mixtures were assumed to be cross-linked completely and the cylinder of composite bone filler was prepared for later experiment.

\subsection{Preparation of extracts from GTF composite}

The GTF composite was shaped into a cylindrical specimen $5 \mathrm{~mm}$ in diamctcr and $0.2 \mathrm{~g}$ in weight. Each GTF composite was placed in a capped plastic test tube with $20 \mathrm{ml}$ normal saline inside. All the test tubes with GTF composite and $20 \mathrm{ml}$ normal saline were kept in an incubator at a temperature of $37{ }^{\circ} \mathrm{C}[19,20]$. After soaking for $1,2,4,6$, and 8 days, the extracts were collected for use in cell cullure, gas chromatography (GC), capillary electrophoresis (CE), and inductive coupled plasma-atomic emission spectrometry (ICP-AES).

\subsection{Analysis of extracts from GTF composite}

It is important to measure the content of possible constituents in extracts, which were released from the 
series of GTF composites soaked in the normal saline for a period of time. In this study, the calcium content in individual extracts was determined by ICP-AES, and the gelatin concentration was measured by $\mathrm{CE}$.

Gas chromatography (GC) was used to detect the amount of uncross-linked or unchained formaldehyde in the series of GTF composites. Two $\mu 1$ of extract from a GTF composite were injected into a 6 inch stainless steel column packed with 50/80 mesh Supelcoport-Porapak $N$ obtained from Supelco, Inc. The helium carrier gas was set at 35 , the oven temperature was $110^{\circ} \mathrm{C}$, the injection temperature and the flame detector temperature were $170^{\circ} \mathrm{C}$. A pure formaldehyde solution containing $37 \%$ formaldehyde was used as a standard, and this solution was diluted with distilled water to prepare a series of standard formaldehyde solutions in the concentrations of $16,12,8,4,2,1$ and $0.5 \%$. Two $\mu 1$ of the series of formaldehyde solutions were injected into the instruments to get the calibration curve.

\subsection{Testing of cytotoxicity of extracts from GTF composite}

The purpose of the testing was to examine the possible influences of GTF extracts on the growth and morphological transformation of human myoblast. The extracts for use in cell cultures were sterilized by filtration through $0.2 \mu \mathrm{m}$ millipore filters and added to cell cultures.

Human myoblasts collected from National Taiwan University Hospital were grown in 24 Linbro well plates or Petri dishes ( $4 \mathrm{~cm}$ in diameter) in Eagle's minimum essential medium (MEM), supplemented with $10 \%$ fetal calf serum and $1 \%$ antibiotics. Cell cultures were maintained in a humidified atmosphere with $5 \%$ $\mathrm{CO}_{2}$ at a temperature of $37^{\circ} \mathrm{C}$. The myoblasts were placed in six-welled tissue culture plates $\left(1 \times 10^{5}\right.$ cells per well) in complete medium and incubated for $24 \mathrm{~h}$ as described. At $24 \mathrm{~h}$, the medium was replaced and extracts from the series of GTF composites were added in a ratio of $1: 1$, giving a final extract concentration of $50 \%[19,23]$. In the control group, the normal saline was mixed with complete medium in a ratio of $1: 1$ for cell culture. After being cultured for 2 days, the medium was removed and the cells were washed twice with phosphate buffer solution (PBS). The cells were trypsinized, resuspended, and then $1 \mathrm{ml}$ trypan blue was added to make sure the cells were still living. They were counted in a Neubauer counting-chamber under an optical microscope [24].

For morphological observation, the medium was removed from the culture dishes. The culture dishes were washed with $0.185 \mathrm{M}$ sodium cacodylate buffer ( $\mathrm{pH}$ 7.2 ) and fixed with $4 \%$ glutaraldehyde solution for 30 min. They were dehydrated through a graded ethanol series and stained with hematoxylin/eosin for optical microscopic examination [23-25].

\section{Results and discussion}

\subsection{The residual formaldehyde in the GTF composites}

Fig. 1 shows the formaldehyde concentration in the extracts after the series of GTF composites had been soaked in the normal saline for $1,2,4,6$, and 8 days, respectively. The curves in Fig. 1 could be divided into two groups. The first group was a low formaldehyde concentration group in a range of approx. $2-6 \mu \mathrm{g} \mathrm{ml}^{-1}$ of formaldehyde in the extracts, which were released by the GTF composites cross-linked with $1,2,4$ and $8 \%$ of formaldehyde. The second group was regarded as a high formaldehyde concentration group, with a concentration of formaldehyde about $4 \mu \mathrm{g} \mathrm{ml}^{-1}(2-10 \mu \mathrm{g}$ $\mathrm{ml}^{-1}$ ) higher than that in the extracts of the first group, which came from the GTF composites cross-linked with 12 and $16 \%$ of formaldehyde. All the curves in Fig. 1 demonstrate a common tendency, whereby the residual formaldehyde released from the various GTF composites reached a plateau after a 4-day soaking period. The tendency reflected the fact that the residual or uncross-linked formaldehyde in the GTF composites would be completely released after the composites had been soaked in the normal saline for 4 days.

The curves corresponding to the low formaldehyde concentration group show no significant difference in formaldehyde concentration in the extracts. However, the curves corrcsponding to the high formaldehyde concentration group exhibit an apparent difference in formaldehyde concentration in the extracts, the $12 \%$ formaldehyde cross-linked GTF composite always showing less formaldehyde released than that of the $16 \%$ formaldehyde cross-linked GTF composite, during the soaking periods. Two points could be summarized

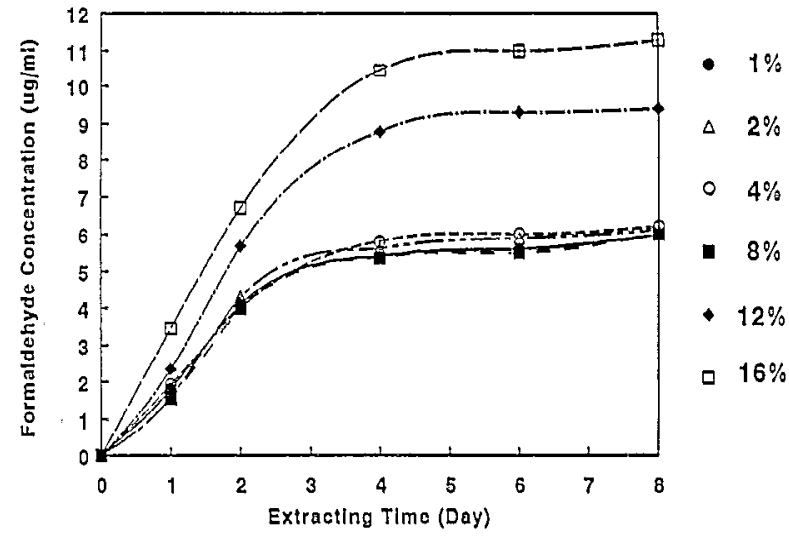

Fig. 1. The formaldehyde concentration in the extracts after the series of GTF composites were soaked in the normal saline for $1,2,4,6$, and 8 days, respectively. 
from the above-mentioned scenario: (1) the formaldehyde cross-linking agent added to the GTF composite should be lower than $8 \%$ in order to prevent the unchained formaldehyde leaking out from the GTF composite; (2) the formaldehyde cross-linked GTF composites should be soaked in the normal saline for at least 4 days before being prepared for cell culture evaluation, in order to guarantee their non-toxicity.

\subsection{The effects of GTF extracts on myoblast cell culture}

The main purpose of the study was to evaluate the toxicity of the GTF composites. The human myoblasts were prepared for evaluation of the series of GTF composites and the blank normal saline was used as a control group. Fig. 2 shows the relationship between cell number and extractive periods after the series of GTF composite extracts cocultured with $1 \times 10^{5}$ human myoblasts in a cultural well for 2 days [24-26]. The cell number decreased with the extractive period in the initial four days, and thereafter increased. In addition, the cell numbers with 6- and 8-day extractive periods were higher than those of the control group where the $1 \times 10^{5}$ human myoblasts cocultured with blank normal saline instead of GTF composite extract. The myoblasts were increasing up to approx. 2.5 -fold $\left(2.5 \times 10^{5}\right)$ of the original amount $\left(1 \times 10^{5}\right)$ after coculturing with blank normal saline for two days. The curve representing $4 \%$ formaldehyde cross-linked GTF composite, for instance, initially decreased with the extractive time, and then began to increase after an extractive time of over 4 days. The cell number was greater than that of the control group, which was almost constant at approx. $2.5 \times 10^{5}$, after being cultured for 2 days.

As described in the previous section, the residual or unchained formaldehyde of the GTF composite was completely released after soaking in normal saline for 4

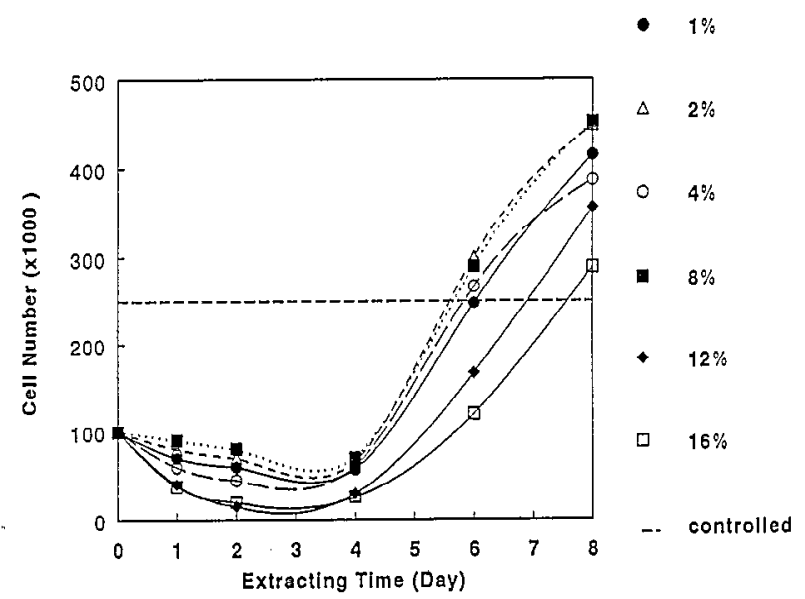

Fig. 2. The relationship between cell number and extractive periods after the series of GTF composite extracts cocultured with $1 \times 10^{5}$ human myoblasts for 2 days. days. Formaldehyde has long been criticized for its toxicity in living tissue $[18,19]$. The authors considered that the growths of myoblasts were inhibited by the residual or unchained formaldehyde, which remained during the initial 4 days after extraction, and this is reflected in Fig. 2. Also in Fig. 2, however, the myoblasts show a progressive growth after coculturing with extracts soaked for over 4 days. It is possible that the GTF composites might release some nutritious constituents after soaking in normal saline for over 4 days. The nutritious constituents might be beneficial to the growth of human myoblast.

The presence of gelatin in the GTF composites serves a two-fold purpose [16,17]: it immobilizes the TCP particles and it improves the mechanical properties. While this composite does not survive long-term in the physiological environment, it is resorbable. The gelatin matrix is resorbed, allowing for new bone intergrowth around the TCP particles [16-19]. The TCP particles are also resorbable, but the resorption rate of these particles is much slower than that of the gelatin matrix. It is controlled by cellular and solution-driven processes. As we learned from the main ingredients of the GTF composites, the possible constituents released from the GTF (except for formaldehyde) were gelatin, calcium ions, and phosphorus ions.

\subsection{The gelatin molecules released from the GTF composites and their effects on the growth of human myoblast}

Fig. 3 shows the concentration of gelatin molecules in the extracts of the series of GTF composites soaked in the normal saline. All the curves in Fig. 3 demonstrate a low gelatin concentration in the extracts, in the initial four days. After the series of GTF composites were soaked in the normal saline for 4-6 days, the gelatin concentration in the extracts rapidly increased, averaging from $0.1 \mathrm{mg} \mathrm{ml}^{-1}$ to $0.6 \mathrm{mg} \mathrm{ml}^{-1}$, respectively. After the series of GTF composites had been soaked for over

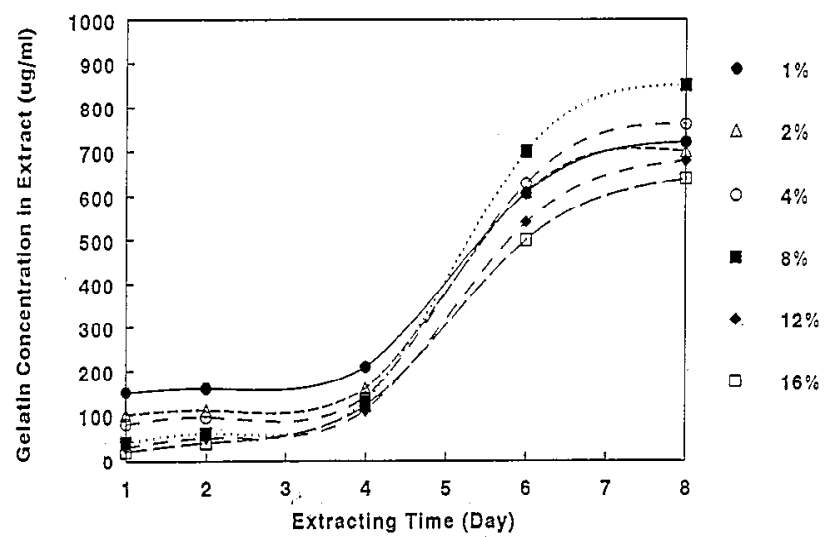

Fig. 3. The gelatin concentration in the extracts of the series of GTF composites soaked in the normal saline for a period of time. 
6 days, the gelatin concentration in the extracts basically reached a constant value of approx. $0.6-0.75 \mathrm{mg} \mathrm{m}^{-1}$.

The results of the gelatin concentration in the extracts implied a relationship between the gelatin release rate and the soaking time of the series of GTF composites. The gelatin showed a slow release rate in the initial 4 days because it was well cross-linked by the formaldehyde. The unchained gelatin was then gradually released from the series of GTF composites, after the latter had been soaked for 4-6 days. The gelatin concentration in the extracts, theoretically, should increase with the soaking time. The curves in Fig. 3, however, turn into a plateau, which indicates that the gelatin concentration was maintained at a constant level. Although gelatin was continuously released from the GTF composite, the gelatin molecules in the extract would be further decomposed into peptides or small amino acid molecules $[20-22,27]$. The new, unchained gelatin was released from the extracts, but the old gelatin molecules decomposed in the extracts, thereby keeping the gelatin concentration in the extracts at a constant value.

The gelatin concentration in the extracts was in the range of $0.05 \mathrm{mg} \mathrm{m}^{-1}-0.8 \mathrm{mg} \mathrm{ml}^{-1}$. What would be the effect of gelatin concentration on the growth of human myoblast? Would it be promoted or inhibited? The authors prepared a series of gelatin solutions in different concentrations of $0 \mathrm{mg} \mathrm{ml}^{-1}-1 \mathrm{mg} \mathrm{ml}^{-1}$, with $0.2 \mathrm{mg} \mathrm{m} \mathrm{m}^{-1}$ increment. The gelatin solutions were cocultured with $1 \times 10^{5}$ human myoblasts in a cultural well for 2 days, and then their cell number and cell morphology were examined. The results indicated that cell number increased in response to the gelatin concentration, as shown in Fig. 4. Fig. 5(a) and 5(b) show the morphology of the human myoblast cocultured with gelatin concentrations of $0 \mathrm{mg} \mathrm{ml}^{-1}$ and $0.8 \mathrm{mg} \mathrm{ml}^{-1}$, respectively. There was no significant difference in the morphology of human myoblast observed under the optical microscope. The results showed that (1) the gelatin would promote the growth of the myoblast and

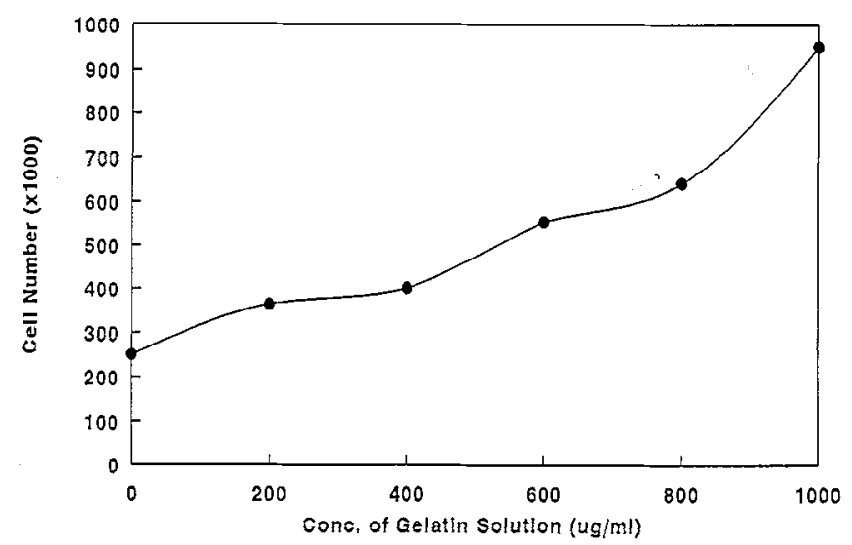

Fig. 4. The effect of gelatin concentration on human myoblast growth after gelatin solutions in different concentrations cocultured with $1 \times 10^{5}$ human myoblasts for 2 days.

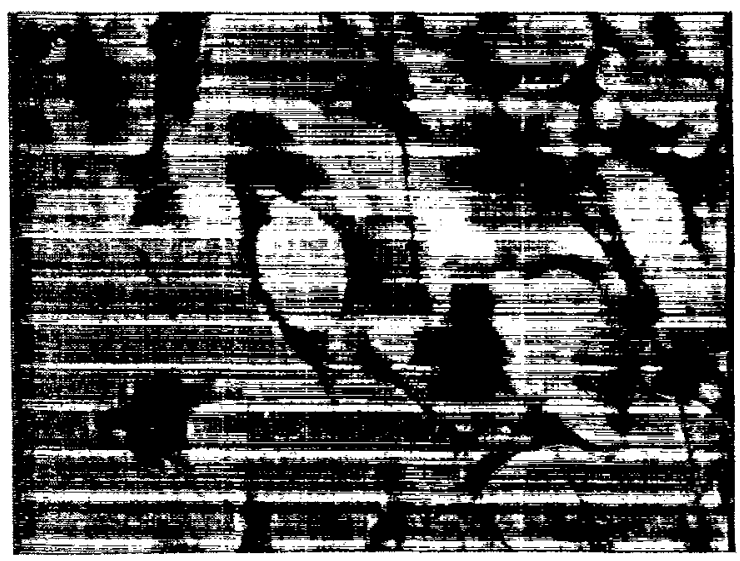

$50 \mu \mathrm{m}$

(a)

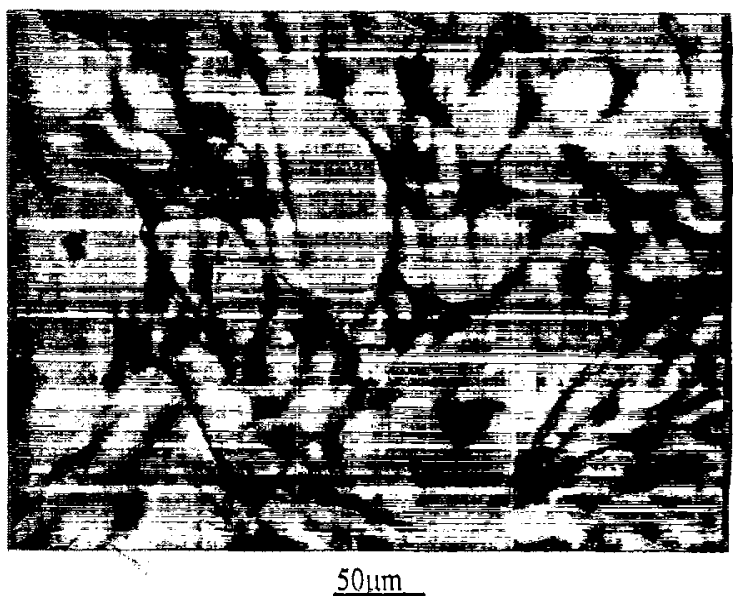

(b)

Fig. 5. The morphology of the human myoblast cocultured with gelatin concentrations of: (a) $0 \mathrm{mg} \mathrm{ml}^{-1}$, and (b) $0.8 \mathrm{mg} \mathrm{ml}^{-1}$ observed under the optical microscope.

could be regarded as one of the nutritious elements favorable to myoblast growth; (2) a gelatin concentration of less than $1 \mathrm{mg} \mathrm{ml}^{-1}$ in the extracts would not induce the morphological transformation of the human myoblast.

The results in this section were coincident with that of Section 3.2. The cell number for the cocultured GTF extracts increased significantly after the series of GTF composites were soaked in the normal saline for over 4 days, which was in agreement with the release of formaldehyde and gelatin from the GTF composites.

\subsection{The calcium ion released from the GTF composites and its effects on the growin of human myoulast}

The measurement of calcium content in the extracts was achieved by ICP-AES. In the process of bone defect healing, the calcium ions play an important role. Calcium ion is a required element in the ensuing ossification process because it is needed to reconstitute the mineral structure of regenerating bone $[2,7]$. This is one 


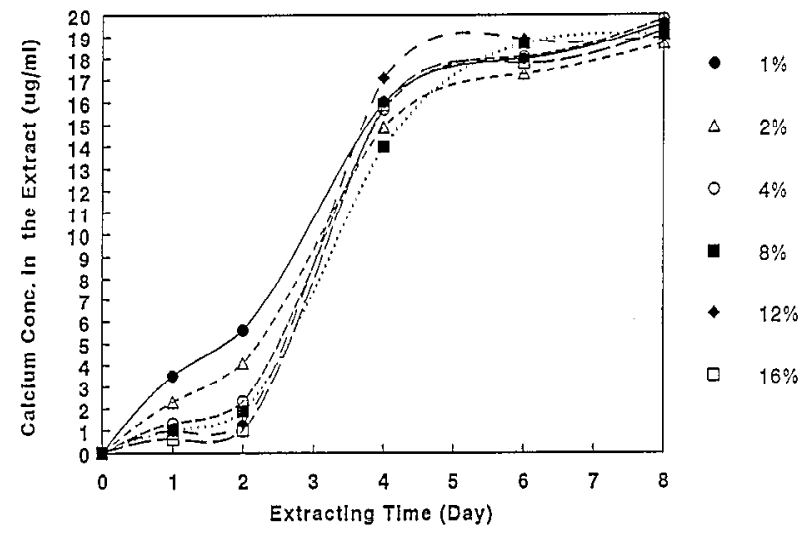

Fig. 6. The relationship between the calcium content in the extracts and the soaking periods of the GTF composites in the normal saline.

of the reasons why we measured the calcium content in the extracts released from the developed material. Fig. 6 shows the connection between the calcium content in the extracts and the soaking periods of GTF composite in normal saline. The curves in Fig. 6 demonstrate that the calcium content in the extracts increased with the soaking periods of the GTF composites. We can observe from Fig. 6 that all the curves are very close in calcium content at each time period. This fact indicated that the calcium content in the extract released from the series of GTF composites had nothing to do with the concentration of formaldehyde, the cross-linking agent for GTF composite. When the series of GTF composites were soaked in the normal saline for 2 days, the calcium content in the extracts was approx. $3 \mu \mathrm{m}$ $\mathrm{ml}^{-1}-5 \mu \mathrm{g} \mathrm{ml} \mathrm{m}^{-1}$. After the series of GTF composites had soaked for over 4 days, the calcium content in the extracts was progressively increasing with the soaking period of the GTF composites. The authors speculated that the calcium ions' presence in the extracts was due to the TCP particles dissolving in the normal saline. In the initial 2 days, the calcium ions in the extracts were attributed to the TCP particles dissolving in the solution. The dissolving TCP particles were thought to be on the free surface of the GTF composites and exposed to the normal saline. After the GTF composites had soaked in the normal saline for over 4 days, the gelatin decomposed and was released from the GTF composites as described in the previous discussion, which would lead to more TCP particles coming into contact with the solution. The unchained gelatin molecules were gradually released from the GTF composite, and then TCP particles kept being exposed/dissolved in the solution, which would explain the constantly increasing calcium content in the extracts.

It has been progressively revealed that minor or trace elements in a biological cell play important roles in various biological processes including metabolism, cell growth, gene expression, and cancer growth $[24,25,28]$.

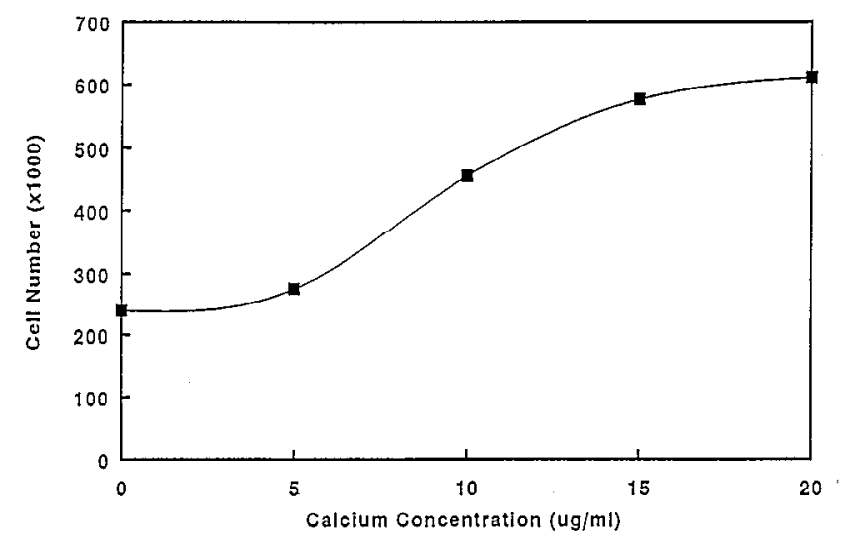

Fig. 7. The effect of calcium content in the solution on human myoblast growth after coculturing for 2 days.

The same question should be posed: what would be the effects of calcium content in the extracts on morphological and functional changes of the myoblast? The authors prepared a series of solutions with a difference in calcium content in a range of $0-20 \mu \mathrm{g} \mathrm{ml}^{-1}$, and then cocultured them with the $1 \times 10^{5}$ myoblasts for 2 days. The results, as shown in Fig. 7 , revealed that the cell numbers increased with the calcium content and the control group (solution without calcium or $0 \mu \mathrm{g} \mathrm{m}^{-1}$ ) had the lowest value. It is possible that the calcium ion in the extracts would be beneficial to the growth of the myoblast. Fig. 8(a) shows the morphology of myoblast colcultured with the solution of $10 \mu \mathrm{g} \mathrm{m} l^{-1}$ calcium content for 1 day. The myoblasts proliferated to confluence and progressive fusion, to form a large multinucleate myocell. However, the myoblast cocultured with the control solution just attached to the well ground and there is no sign of a fusion process having occurred (Fig. $8(b))$. It is easily seen in the cell growing in solution with $10 \mu \mathrm{g} \mathrm{ml}^{-1}$ calcium content for 2 days (Fig. 9(a)). The myoblasts were progressively fused together and form a group of parallel myofibril as a scaled-down model of the myofibrilla. On the other hand, the morphology of the cell cocultured with the control solution showed only a few multinucleated cells (fusion) sporadically spreading around the cultural dish (Fig. 9(b)). There is no fibril-like structure to be observed under the optical microscopic examination.

Muscle cells have been useful for studies of cellular differentiation, since morphological and functional changes occurring during myogenesis were well characterized and could be observed in tissue culture $[27,28]$. Differentiation of the L8 rat skeleton muscle line was marked by myoblast proliferation to confluence, followed by progressive fusion to large multinucleate syncytia, which synthesize muscle-specific proteins and contract spontaneously [28,29]. Although the sequence of functional changes was well defined, the triggering events and control mechanisms underlying the transi- 


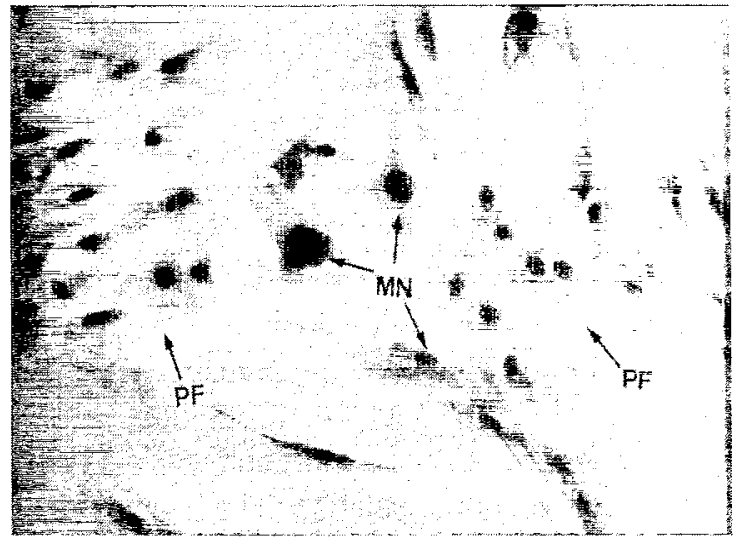

$\frac{50 \mu \mathrm{m}}{\text { (a) }}$

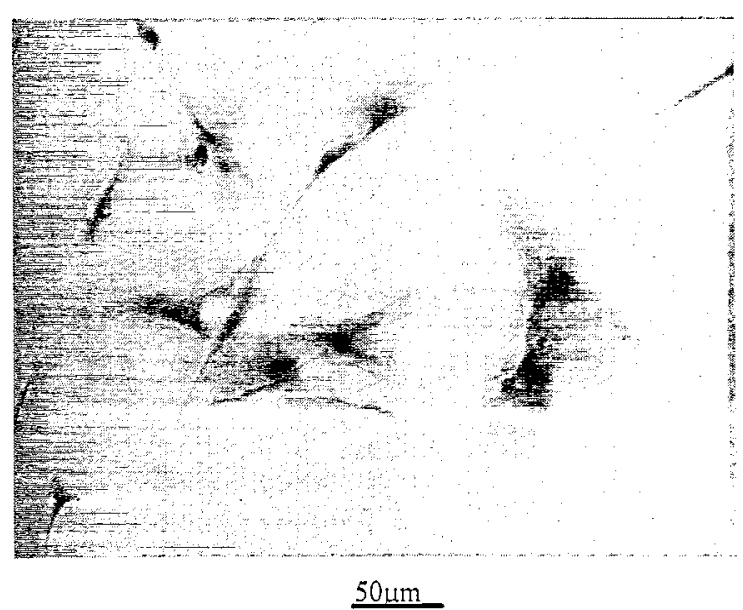

(b)

Fig. 8. The morphology of human myoblast cocultured with a solution of: (a) $10 \mu \mathrm{g} \mathrm{ml}^{-1}$, and (b) $0 \mu \mathrm{g} \mathrm{ml}^{-1}$ calcium content for 1 day. MN: multinucleate myoblast, PF: progressive fusion.

tion from relatively undifferentiated myoblast to specialized muscle cell were poorly understood. Knudsen et al. [25] had reported that mature skeleton muscle cells were distinguished by being multinucleate, a state resulting from the fusion of myoblasts and occurring only in the presence of physiological concentrations of extracellular $\mathrm{Ca}^{2+}$. In this experiment, the calcium ion might be beneficial to the growth of the myoblast and would promote premature growth of the latter, thereby encouraging cell fusion or early development of the fibrilla structure.

Finally, we could observe the myoblasts cocultured with the extracts of $4 \%$ formaldehyde cross-linked GTF composite for 2 days (Fig. 10(a)). The myoblasts are well-attached, confluent, and fused in parallel with each other like small sized muscle fiber. The myoblast cultured with the normal medium, with $10 \%$ fetus and antibiotics added, is shown in (Fig. 10(b)). Although the cell also shows a well-attached condition, there is no fibrous structure to be seen and fusion is only in an

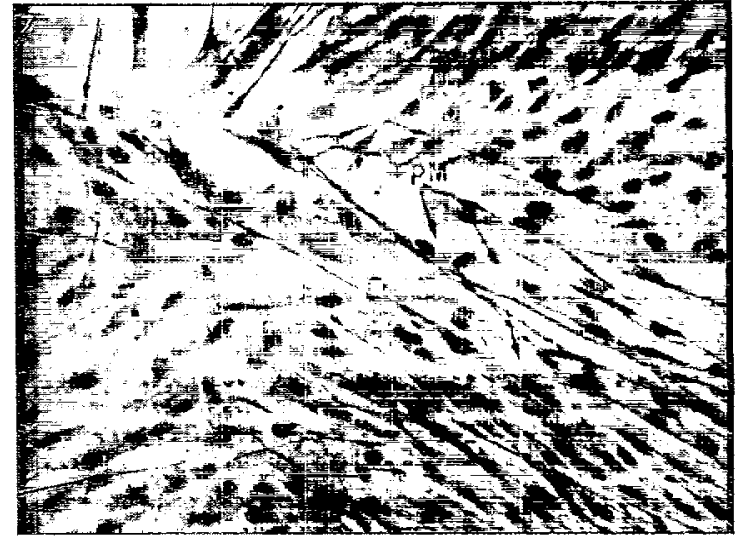

$\underline{504 \mathrm{~m}}$

(a)

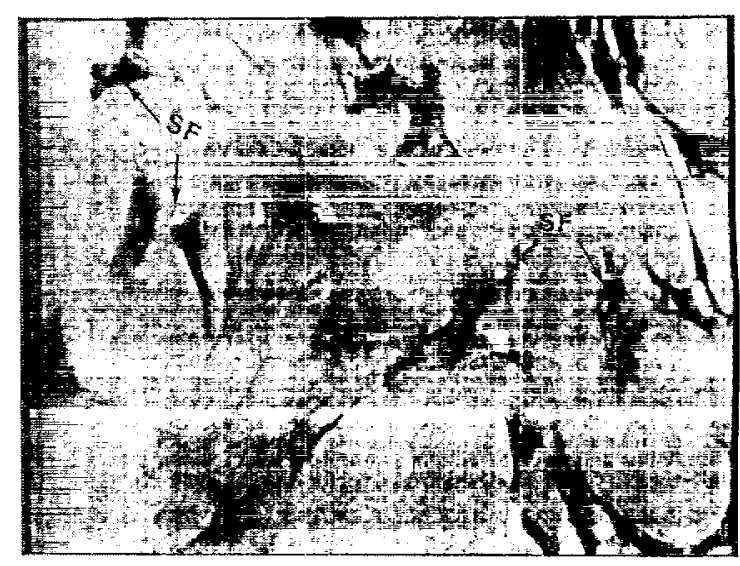

$\frac{50 \mathrm{um}}{(\mathrm{b})}$

Fig. 9. The morphology of human myoblast cocultured with a solution of: (a) $10 \mu \mathrm{g} \mathrm{m} \mathrm{l}^{-1}$, and (b) $0 \mu \mathrm{g} \mathrm{mi}-1$ calcium content for 2 days. SF: sporadic fusion, PM: parallel myofibril-like structure.

initial stage $[26,30]$. The observation supports the view that the GTF composite would not inhibit the myoblast growth and would not cause morphological transformation.

\section{Conclusions}

The developed GTF composites cross-linked with formaldehyde in a concentration lower than $8 \%$ showed no toxicity to the human myoblast. They also did not change the morphology or functions of the myoblast. The unchained or uncross-linked formaldehyde in the series of GTF composites would be completely released after soaking in the normal saline for 4 days. Cell numbers in the myoblasts cocultured with extracts were lower than in those of the control group in the initial 4-day soaking period. However, the cell numbers rapidly increased and outnumbered those of the control group after the myoblasts cocultured with the extracts of GTF 


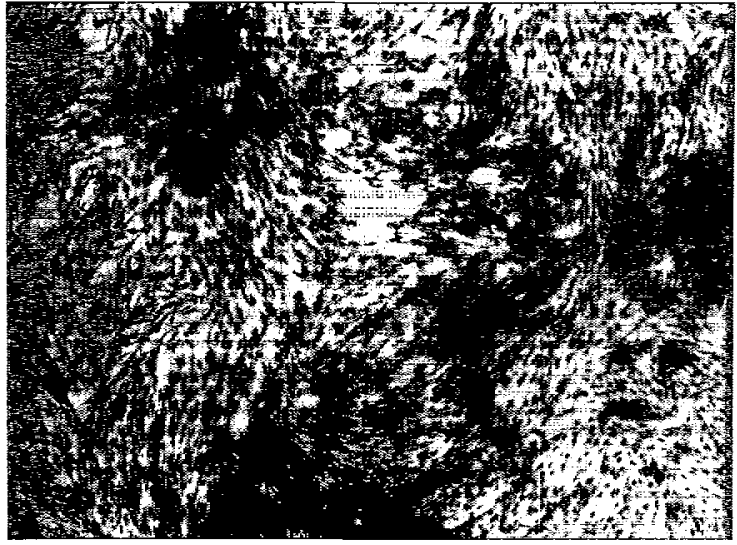

$\underline{200 u m}$

(a)

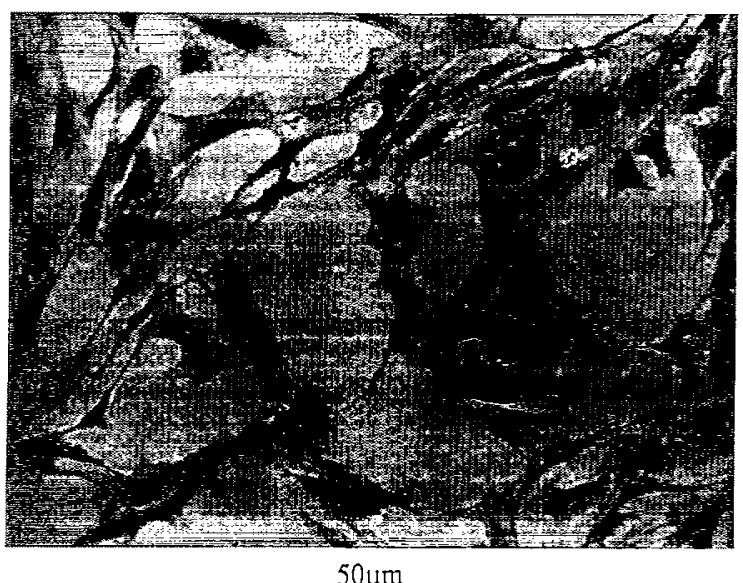

(b)

Fig. 10. (a) The morphology of the human myoblast cocultured with the 6-day extract of $4 \%$ formaldehyde cross-linked GTF composite for 2 days. (b) The morphology of the human myoblast cocultured with the normal medium, with $10 \%$ fetus and antibiotics added, for 2 days.

composite soaked for over 4-6 days. The authors speculated that the myoblast growth was initially inhibited by the unchained formaldehyde released from the GTF composite. After extracts of the GTF composite were soaked in normal saline for 4-6 days, calcium ions and gelatin were gradually released from the GTF composite and were beneficial to the growth of the myoblast. The GTF composite is thought to be an excellent bioabsorbed bone graft and has a great potential for orthopedic applications.

\section{Acknowledgments}

The authors would sincerely like to thank the National Science Council (ROC) for their financial support with the grant of NSC84-0412-B-002-72-M08. We dedicate this paper with gratitude to the National Science Council (ROC).

\section{References}

[1] M.R. Urist, Bone Grafts, Derivatives and Substitutes, CRC Press, Boca Raton, Florida, 1994.

[2] R.W. Bucholz, A. Carlton and R.E. Holmes, Hydroxyapatite and tricalcium phosphate bone graft substitutes, Orthop. Clin. North Am., 18 (1987) 323.

[3] S.L. Turek, Orthopedics Principles and their Application, 4th edn., 1984, pp. 31-100.

[4] C.F. Lord, M.C. Gebhardt, W.W. Tomford and H.J. Mankin, Infection in bone allografts, J. Bone Jt. Surg., 70A (1988) $369-376$.

[5] B.H. Berry, C.F. Lord, M.C. Gebhardt and H.J. Mankin, Allograft fractures: frequency, treatment, and end results, $J$. Bone Jt. Surg., 72A (1989) 825-833.

[6] M. Aebi, Bone Transplantation, Updating One Osteochondral Auto and Allografting, Springer, Berlin, 1987.

[7] F.H. Lin, C.C. Liu, Y.Y. Huang, C.Y. Wang and C.M. Lu, Sintered porous DP-bioactive glass and hydroxyapatite as bone substitute, J. Biomater., 15 (1994) 1087-1097.

[8] J.W. Harrison, Osteosarcoma associated with metallic implant, Clin. Orthop. Relat. Res., 116 (1976) 253-260.

[9] R. Holmes, R. Bucholz and V. Mooney, Porous hydroxyapatite as a bone-graft substitute in metaphyseal defects, a histometric study, J. Bone Jt. Surg., 68A (1986) 904.

[10] F.H. Lin, Y.Y. Huang, M.H. Hon and S.C. Wu, Fabrication and biocompatibility of a porous bioglass ceramic in a $\mathrm{Na}_{2} \mathrm{O}-\mathrm{CaO}-\mathrm{SiO}_{2}-\mathrm{P}_{2} \mathrm{O}_{5}$ system, J. Biomed. Eng., 13 (1991) $328-334$.

[11] J.F. Piecuch, A.J. Goldberg, C.V. Shastry and R.B. Chrzanowski, Compressive strength of implanted porous replamine form hydroxyapatite, J. Biomed. Mater. Res., 18 (1984) 39-45.

[12] N. Blumenthal, T. Sabe and E. Barrington, Healing responses to grafting of combined collagen-decalcified bone in periodontal defects in dogs, J. Periodontol, 57 (1986) 84-88.

[13] M. Minabe, A. Sugaya, H. Satou, T. Tamura, Y. Ogawa, T. Hari and Y. Watanbe, Histological study of the hydroxyapatitecollagen complex implants in periodontal osseous defects in dogs, J. Periodontol., 59 (1988) 671-678.

[14] K. Ono, T. Yammamuro, T. Nakamura, Y. Kakutani and T. Kokubo, Apatite-wollastonite containing glass ceramic-fibrin mixtures as a bone defect filler, J. Biomed. Mater. Res., 22 (1988) 869-885.

[15] C.C.P.M. Verheyen, J.R. de Wijin, C.A. van Blitterswijk, K. de Groot and P.M. Rozing, Hydroxyapatite/poly (L-lactide) composites: an animal study on push-out strengths and interface histology, J. Biomed. Mater. Res., 27 (1993) 433-444.

[16] K.S. Tenhuisen and P.W. Brown, The formation of hydroxyapatite-gelatin composite at $38^{\circ} \mathrm{C}, \mathrm{J}$. Biomed. Mater. Res., 28 (1994) $27-33$.

[17] J.O. Hollinger and G.C. Battistone, Biodegradable bone repair materials, Clin. Orthop. Relat. Res., 207 (1986) 290-305.

[18] D.F. Williams, Biocompatibility of Clinical Implant Materials, Vol. 2, 9 (1981) 209-234.

[19] H.E. Koehlein and G. Lemperle, Studies of gelatin-resorcinformaldehyde glue, Surgery, 66 (1969) 377-383.

[20] D.P. Speer, M. Chvapil, C.D. Eskelson and J. Ulreich, Biological effects of residual glutaraldehyde in glutaraldehyde-tanned collagen biomaterials, J. Biomed. Mater. Res., 14 (1980) 753764.

[21] W.C. McMaster, J. Kouzelos, S. Liddls and T.R. Waugh, Tendon grafting with glutaraldehyde fixed material, J. Biomed. Mater. Res., 10 (1976) 259-271.

[22] M. Vert, P. Christel, F. Chabot and J. Leary, Bioresorbable plastic materials for bone surgery, in Macromolecular Biomaterials, CRC Press, Boca Raton, Florida, 1984.

[23] S.F. Konieczny, J. McKay and J.R. Coleman, Isolation and 
characterization of terminally differentiation chicken and rat skeletal muscle myoblasts, Dev. Biol, 91 (1982) 11-26.

[24] C. Derker, R. Greggs, K. Duggan, J. Stubbs and A. Horwitz, Adhesive multiplicity in the interaction of embryonic fibrolasts and myoblasts with extracellular matrices, Cell Biol., 99 (1984) $1398-1404$.

[25] K.A. Knudsen, L. Myers and S.A. McElwee, A role for the $\mathrm{Ca}^{+2}$-dependent adhesion molecule, $\mathrm{N}$-cadherin, in myoblast interaction during myogenesis, Exp. Cell. Res., 188 (1990) 175184.

[26] T. Endo and B. Nadal-Ginard, Three types of muscle-specific gene expression in fusion-blocked rat skeleton muscle cells: translation control in EGTA-treated cells, Cell, 49 (1987) 515528.

[27] C.H. Yao, Y.L. Chang and F.H. Lin, Preparation and evaluation of B-TCP powder and cross-linked gelatin composite as bone substitute, Chinese Med. Bio. Eng., 14 (1) (1994) 47-51.

[28] H.U. Blau and C.J. Epstein, Manipulation of myogenesis in vitro: reversible inhibition by DMSO, Cell, 17 (1979) 95-108.

[29] C. Richler and D. Yaffe, The in-vitro cultivation and differentiation capacities of myogenic cell, Dev. Biol., 23 (1970) 1-22.

[30] S.J. Raufman, C.M. Parks, J. Bohr and L.E. Faiman, Transformation is an alternative to normal skeleton muscle development, Exp. Cell Res., 125 (1980) 333-349. 